\title{
The Effect of Swimming Training on Serum Levels of Sex Hormones, Antioxidant Enzymes and Oxidative Damage of DNA Ovarian Tissue in Rats with Polycyclic Ovarian Syndrome
}

\author{
${ }^{1}$ Mehdi Noura*, ${ }^{2}$ Samane Rafie, ${ }^{1}$ Seyed Ali Hosseini, ${ }^{3}$ Mohammad Amin Edalat Manesh \\ ${ }^{1}$ Department of Sport Physiology, Marvdasht Branch, Islamic Azad University, Marvdasht, Iran. \\ ${ }^{2}$ Department of Sport Physiology, Kish International Paradise, University of Tehran, Kish, Iran. \\ ${ }^{3}$ Department of Physiology, Science Faculty, Shiraz Branch, Islamic Azad University, Shiraz, Iran.
}

Submitted 02 March 2020; Accepted in final form 15 May 2020.

\begin{abstract}
Background. Hormone disorders, stress-oxidative and inflammation in ovarian tissue cause no- ovulation in women with polycyclic ovarian syndrome (PCOS). Several studies have shown the role of exercises in control of sex hormones. Objectives. The aim of this study was to investigate the effect of 6 weeks of swimming training (ST) on serum levels of sex hormones, antioxidant enzymes and oxidative damage of DNA ovarian tissue in rats with PCOS. Methods. In this experimental study, 30 female Wistar rats were divided into 3 groups included control, PCO (letrozole consumption for 28 days intra- peritoneally) and PCO+ST groups. PCOS was induced with 28 days of peritoneal injection of $1 \mathrm{mg} / \mathrm{kg}$ letrozole. The ST program was included 6 weeks of ST for 20 minutes per day. At the end of the treatment period, serum levels of LH, FSH, testosterone, estradiol and progesterone, SOD, CAT, GPX, MDA and HOdG-8 in ovarian tissue were measured by ELISA method. Results. ST significantly decreased serum levels of LH, estradiol, testosterone, MDA and HOdG-8 levels $(\mathrm{p}<0.05)$ as well as increased SOD, CAT, and GPX enzymes of rats with PCOS $(\mathrm{p}<0.05)$. Conclusion. It appears that ST has antioxidant and sex hormone enhancing effects in ovarian tissue of rats with PCOS model.
\end{abstract}

KEYWORDS: Polycystic Ovarian Syndrome, Training, Sex Hormones, Oxidative Stress.

\section{INTRODUCTION}

Polycystic ovary syndrome (PCOS) is a common endocrine disorder which affecting about $5-10 \%$ of women with premenopausal women (1). Endocrine disorders in these women include no- ovulation or limited ovulation, infertility, and acne, which increase the biology levels of testosterone and increase the production of androgens from the ovaries (2). The polycystic ovary is the result of a functional disorder (3). A range of abnormalities that cause no- ovulation is associated with impaired hypothalamic-pituitarygonadal axis. However, many of these disruptions are temporary. PCOS reflects a persistent and prolonged ovarian failure which in turn causes a uniform condition resulting from estrogen feedback on the secretion of gonadotropin and the level of follicular stimulatory hormone is reduced to low levels in the normal range. In addition, there is no peak of its secretion in the middle of the cycle. The secretion of the luteinizing hormone is maintained at an elevated level, but does not have an appropriate increase in mid-cycle. Initially, the constant stimulation of gonadotropin secretion causes follicles to grow and estradiol and estrogen

*. Corresponding Author:

Mehdi Noura, Assistant Professor

E-mail: noora.mehdi@yahoo.com 
increase, but the oocytes is not released. After a while, the growth of the follicles stops and the follicles become cystic and atrophic (4). The increase in androgens affects the formation and release of oocyte during oviposition and causes the development of a large number of follicles. Regarding the fact that in patients with PCOS, the growth of follicles in the phase of the selection of dominant follicle is stopped and cystic follicles formed under the thick capsule of the ovary (5). Research has shown that oxidative stress is a form of imbalance between production and inhibition of free radicals by the antioxidant defense system. Oxidative stress is effective in increasing the production of androgens, disrupting the developmental stages of ovarian follicles and ovarian tissue damage in patients with PCOS. Disorders in synthesis of ovarian steroids have also been reported to be one of the causes of oxidative stress in these patients (6). Studies have shown that serum levels of oxidative stress indices such as reactive oxygen species (ROS) in patients with PCOS increased and total antioxidant capacity (TAC) of blood decreases (7). On the other hand, abnormal increase in lipid peroxidation causes damage to the membrane and cellular organs. Malondialdehyde (MDA) is the final product of peroxidation of fatty acids, and evaluation of the MDA as lipid peroxidation index has particular importance in determining the amount of free radicals (8). Research has shown that guanine is more potent for oxidation than among purine and pyrimidine. As a result of the hydroxyl radicals attacking the eighth position of the guanine molecule, a compound called the 8-hydroxy-doxyguanosine (8-Oxo-2'-deoxyguanosine: 8 -OHdG) is produced. Considering that $8-\mathrm{OHdG}$ shows a dynamic balance between the oxidative damage of DNA and its repair speed, the measurement of this compound is important in evaluating DNA damage (9). Lifestyle modification through dietary adjustment and training programs aimed at normalizing androgen levels and making ovulation is the first line treatment for PCOS. Exercises involving the larger muscles can reduce insulin resistance and is an important part of nonpharmacological treatments. Even in women who have a high genetic predisposition for PCOS, they can control or prevent the onset of the disease through proper lifestyle (10). Research has shown that endurance trainings increase the level of the brain-derived neurotrophic factor (BDNF) of the hippocampus in the tri-methyltine chloride poisoning model (11). Regarding the effects of immune and hormone-modifying effects of physical trainings, the aim of this study was to determine the effect of swimming training (ST) on serum levels of sex hormones, antioxidant enzymes and oxidative damage of DNA ovarian tissue in rats with POCS.

\section{MATERIALS AND METHODS}

In this experimental study, 30 female Sprague Dawley rats were used with an average weight of $200 \pm 10 \mathrm{~g}$. The animals were cultivated at the Shiraz Islamic Azad University and maintained under standard temperature $\left(25 \pm 2^{\circ} \mathrm{C}\right)$ and humidity $(50 \pm 10 \%)$ and 12 hours light and dark cycle (11). Experiments were carried out at least 10 days after the establishment of the animals in order to obtain a compromise with the environment. All stages of animal work were carried out according to the rules of animal protection and supervised by the Ethics Committee of Islamic Azad University of Shiraz with code of IR.IAUSHIRAZ.2543.95.464. All surgical procedures and sampling were performed under full anesthesia and also tried to use the lowest number of acceptable samples.

Animals were randomly divided into 3 groups including: 1) Healthy control (animals of this group did not have any treatment and were used for biochemical studies with other groups), 2) PCO (animals of this group received intra- peritoneal injection of daily $1 \mathrm{mg} / \mathrm{kg}$ letrozole (Letrax, AbuAryhan Pharmaceutical Company, Iran) for 28 days) and 3) PCO + ST (this group performed ST for 6 weeks after induction of PCOS).

To conduct this study, initially animals were selected from 2 to 3 regular courses of estrus during 12 to 14 days of visit of vaginal smear. Vaginal smear was used to determine the regularity of the estrus cycle. First, $0.3 \mathrm{ml}$ of physiological serum was injected slowly into the vagina by the sampler (Brand, Germany). Then take 1 to 2 drops of fluid and smear applied. The samples were examined by optical microscope CX21FS1 (Olympus, Japan). Rats that were in the estrus period of reproductive cycle were selected for the next stages of the study. The vaginal smear in the estrus stage has more horn cells than the epithelial cells and does not have leukocyte (12). Intraperitoneal injection of letrozole for 28 days induces PCOS in rats (11). At the onset of letrozole 
injection, regular ovarian cycles were observed, but gradually estrous cycles became irregular. The irregularity of estrus cycles and the occurrence of a persistent vaginal cornification phase (PVC) is one of the symptoms of follicular cysts in the ovaries (8). The presence of horn cells and the lack of cellular changes (spherical and congested cells) indicate irregularity of ovarian cycles that occurred 28 days after the administration of Letrozole in randomly selected samples. After the end of 28 days of daily administration of $1 \mathrm{mg}$ of Letrozole, several animals were randomly selected and, after being sacrificed with a lethal dose of chloroform (Merck, Germany), the ovaries were removed. After fixation with $10 \%$ formalin, tissue sections from different parts of the ovary were prepared. Colorimetry was performed using hematoxylineosin, and then the ovarian cysts were examined under an optic microscope.

In the $\mathrm{PCO}+\mathrm{ST}$ group, the training protocol included 6 weeks of ST at a rodent pool with 1 meter diameter and with a water temperature of $25 \pm 2{ }^{\circ} \mathrm{C}$ for 20 minutes per session and three sessions per week. After completing the training sessions all rats dried and transferred to the cage.
Forty- eight hours after last training session rats were anesthetized with diethyl ether and blood samples gathered (13) also the ovary was removed from the body of rats.

Serum levels of LH, FSH, beta- estradiol, testosterone and progesterone measured by ELISA methods with ELISA reader machine model 2100 (Stat Fax, USA) and ELISA kits (Abcam, China). Ovarian levels of superoxide dismutase (SOD), catalase (CAT) and glutathione peroxidase (GPX), MDA, and 8-OHdG measured by ELISA methods with ELISA reader machine model 2100 (Stat Fax, USA) and ELISA kits (Fine Test Co., China). For statistical analysis of data Shapiro-Wilk, one-way ANOVA and Tukey's post- hoc tests were used $(\mathrm{p}<0.05)$.

\section{RESULTS}

Proof of the Model. Histopathological studies revealed the presence of cystic follicles in ovarian samples following 28 days of letrozole administration in the PCO group compared to the control and PCO+ST groups (Figure 1). Pathological examinations in this study were performed to examine the PCO syndrome model.
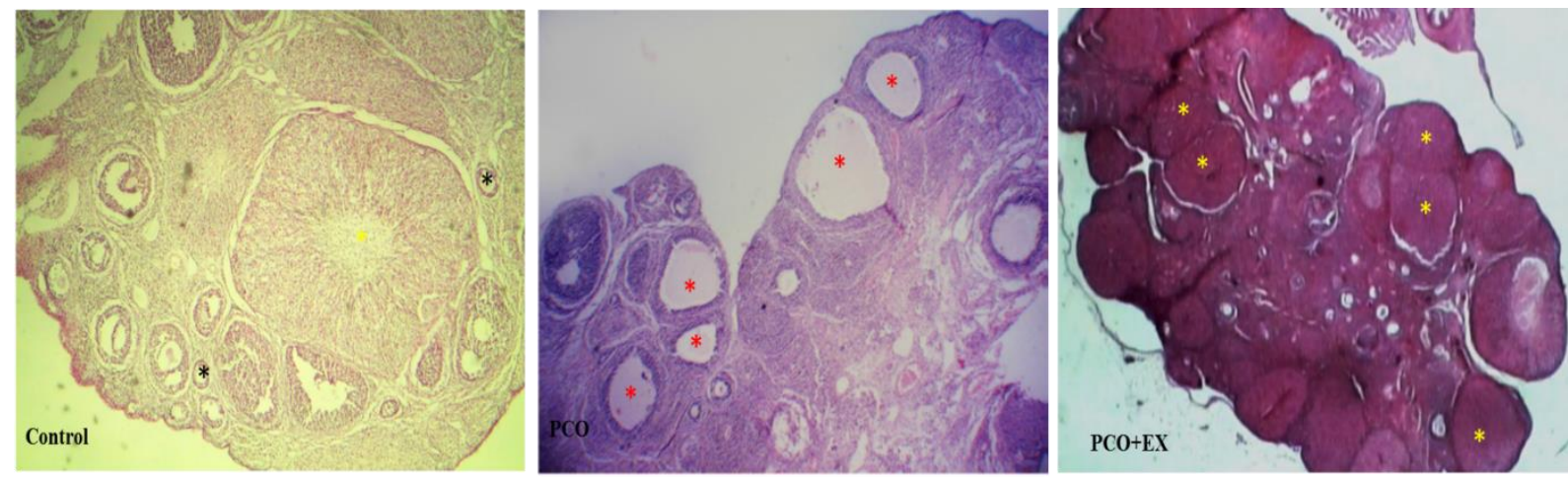

Figure 1. The Photomicrographs of Ovarian Tissue in the Groups. Ovaries in the Control Group Had Normal Phenotype with Normal Primary Follicles. The Cystic Follicles Were Seen in the PCO Group. Increasing Number of Corpus Luteum

Was Seen in the PCO+ST Group Compared to the PCO Group. Dark Star, Pre- Antral Follicles; Red Star, Cystic Follicles; Yellow Star, Corpus Luteum. Hematoxylin-Eosin (H\&E) Staining, 40X Magnification

Antioxidant Activity Analysis. The results showed that the levels of SOD, CAT, GPX enzymes of ovarian tissue in PCO group significantly decreased compare to control group nevertheless the levels of MDA and $8-\mathrm{OHdG}$ significantly increased $(\mathrm{p}<0.05)$. Also levels of SOD, CAT, and GPX in ovarian tissue of PCO+ST group significantly increased as well as levels of MDA and 8-OHdG significantly decreased compared to PCO group $(\mathrm{p}<0.05)$ (Table 1$)$.
Serum Levels of Sex Hormones. Results showed that serum levels of LH, FSH, betaestradiol and testosterone significantly increased and progesterone hormone significantly decreased in PCO group compared to the control group $(\mathrm{p}<0.05)$; also serum levels of $\mathrm{LH}$, beta-estradiol and progesterone significantly decreased in the $\mathrm{PCO}+\mathrm{ST}$ group compared to the PCO group $(\mathrm{p}<0.05)($ Table 2). 
Table 1. Antioxidant Enzymes, MDA and 8-OHdG Levels of Ovarian Tissue in Research Groups

\begin{tabular}{lccccc}
\hline Variable Group & SOD $(\mathbf{p g} / \mathbf{m l})$ & CAT $(\mathbf{m I U} / \mathbf{m l})$ & GPX $(\mathbf{p g} / \mathbf{m l})$ & MDA $(\mathbf{n g} / \mathbf{m l})$ & 8-OHdG $(\mathbf{n g} / \mathbf{m l})$ \\
\hline Control & $69.35 \pm 2.68$ & $138.36 \pm 9.73$ & $95.32 \pm 7.57$ & $32.54 \pm 4.14$ & $5.11 \pm 0.73$ \\
PCO & $21.73 \pm 2.93^{\text {a }}$ & $54.57 \pm 8.45^{\mathrm{a}}$ & $41.48 \pm 6.45^{\text {a }}$ & $81.46 \pm 8.43^{\mathrm{a}}$ & $25.46 \pm 2.43^{\mathrm{a}}$ \\
PCO+EX & $46.32 \pm 4.86^{\mathrm{b}}$ & $68.94 \pm 9.60^{\text {a b }}$ & $51.48 \pm 6.40^{\text {a b }}$ & $65.71 \pm 7.05^{\text {a b }}$ & $10.27 \pm 2.63^{\mathrm{b}}$ \\
\hline
\end{tabular}

Data are presented as Mean \pm SEM; ${ }^{a} P<0.05$ compared with the control group, ${ }^{b} P<0.05$ compared to the $P C O$ group. SOD, Superoxide Dismutase; CAT, Catalase; GPX, Glutathione Peroxidase; MDA, Malondialdehyde; 8-OHdG, 8Hydroxy-Doxy Guanosine.

\begin{tabular}{lccccc}
\multicolumn{7}{c}{ Table 2. Serum Levels of Sex Hormones in Research Groups } \\
\hline $\begin{array}{l}\text { Variable } \\
\text { Group }\end{array}$ & LH $(\mathbf{m I U} / \mathbf{l})$ & $\begin{array}{c}\text { FSH } \\
(\mathbf{m I U} / \mathbf{l})\end{array}$ & $\begin{array}{c}\text { beta-estradiol } \\
(\mathbf{p g} / \mathbf{m l})\end{array}$ & $\begin{array}{c}\text { Progesterone }(\mathbf{p g} / \mathbf{m l}) \\
\text { Testosterone }(\mathrm{pg} / \mathbf{m l})\end{array}$ \\
\hline Control & $2.01 \pm 0.42$ & $3.47 \pm 1.25$ & $22.18 \pm 2.21$ & $20.43 \pm 2.21$ & $0.66 \pm 0.02$ \\
PCO & $7.23 \pm 1.51^{\mathrm{a}}$ & $5.78 \pm 1.30$ & $28.03 \pm 2.23^{\mathrm{a}}$ & $12.74 \pm 1.23^{\mathrm{a}}$ & $1.42 \pm 0.23^{\mathrm{a}}$ \\
& & $\mathrm{a}$ & & & \\
PCO+EX & $3.35 \pm 1.87^{\mathrm{b}}$ & $4.06 \pm 1.19$ & $17.89 \pm 2.51^{\mathrm{b}}$ & $20.80 \pm 3.51^{\mathrm{b}}$ & $0.74 \pm 0.05^{\mathrm{b}}$ \\
\hline
\end{tabular}

Data are presented as Mean \pm SEM; ${ }^{a} \mathrm{P}<0.05$ compared with the control group, ${ }^{b} \mathrm{P}<0.05$ compared to the $\mathrm{PCO}$ group.

\section{DISCUSSION}

Present study investigated the effect of 6 weeks of ST on sex hormones, antioxidant enzymes of SOD, CAT, GPX in ovarian tissue, the amount of DNA oxidative damage and lipid peroxidation in rats with PCOS model. Tissue examination showed that 28 days of $1 \mathrm{mg} / \mathrm{kg}$ letrozole administration could produce ovarian cysts with different dimensions. This finding has been confirmed in previous studies (11). The present study showed that the activity of the antioxidant enzymes of ovarian tissue in rats with PCOS was significantly decreased and the lipid peroxidation as well as oxidative damage of DNA significantly increased in comparison with the control group. It has shown that induction of PCOS induces oxidative stress in ovarian tissue of rats. Also, induction of PCOS by estradiol valerate can increase ovarian cysts by increasing the reactive oxygen species and decreasing the activity of the antioxidant enzymes of the ovary tissue (14). In patients with PCOS, serum levels of oxidative stress indices such as total antioxidant capacity, ROS and radical hydroxyl species are abnormal, and over expression of oxidative stress can lead to reduction of oocyte by mesenchymal hyperplasia of ovary and impairment of its function. (15). Scientific evidences suggest that activity of antioxidant enzymes of ovary tissue decrease in patients with PCOS, and resulting in increased oxidative stress conditions, ovarian androgens increase, and disruption occurs in the process of development of follicular bands (16). Another study found that levels of blood lipids such as triglyceride and oxidized lipid levels in patients with PCOS are high. It has also been reported that as a result of weakening of the antioxidant defense system in these patients, body lipids are not protected against oxidation. Therefore, due to increased blood lipids concentration and weakening of the antioxidant defense system, the chance of oxidative lipid damage has increased and an increase in serum levels of MDA appears (16). It showed that increasing oxidative stress in the ovaries of rats with PCOS causes ovary epithelium DNA damage and granulosic cell apoptosis by increased oxidative stress caused by ROS (17), which is consistent with the results of present study. Women with PCOS have metabolic abnormalities in controlling the production of androgens (18). The high concentrations of androgenic hormones such as testosterone, androstenedione, and dehydroepiandrosterone sulfate have been observed in these patients (19). In PCOS, regular reproductive cycles may be seen without any indication of hyperandrogenism. Although this happens very rarely, most scientists believe that hyperandrogenism should be considered as one of the criteria for defining this syndrome (20).

Studies have shown that exercises can be useful in treating patients with PCOS. Increased physical activities are recommended for people with PCOS. However, there is still insufficient understanding of the amount of exercise, type, and duration of these activities in relation to these patients (21). It has been shown that quality of life is effective on endocrine parameters and Freeman Galvay (FG) scoring scale (22). Combined and interactive effects of exercise and diet improve the level of FSH, sexually transmitted globulin (SHBG), total testosterone, androstenedione and free androgens (23). Wu et al. (2014) studied the effect of exercise in rats with PCOS and showed that serum levels of 
estrogen and testosterone in rats with PCOS were significantly higher than healthy rats (24). Like present study, in $\mathrm{Wu}$ et al. study the trainings have been able to improve the serum levels of androgenic hormones. Another study has shown that metformin consumption and a period of trainings can reduce testosterone, reduce the ratio of hormone luteinizing to follicular stimulatory hormone and improve the metabolic status by weight loss and reducing body mass index; and prevent increase of prolactin, as well as protect from harmful effects of hyperandrogenism (25). Due to changes in weight and sex hormones (free testosterone and androstenedione), trainings especially with low intensity, may improve the symptoms of PCOS (26).

\section{CONCLUSION}

According to results of present study it appears that ST alters the serum levels of sex hormones in the rats with PCOS. Also, ST increases the activity of antioxidant enzymes, decrease the oxidative damage of DNA and lipid peroxidation in ovary tissue in rats with PCOS; so, can concluded that ST can be useful for decreasing some complications of PCOS.

\section{APPLICABLE REMARKS}

- According to results of present study it is suggested that in PCO situation, use swimming training to improve sex hormones.

- Regarding to antioxidant effect of swimming training in present study it is supposed that use swimming training to minimize PCO- induced oxidative stress.

\section{ETHICAL AND LEGAL CONSIDERATIONS}

Researchers received introduction letters from Ethical Committee of Shiraz Branch of Islamic Azad University.

\section{CONFLICTS OF INTEREST}

The authors declare that there is no conflict of interest.

\section{AUTHORS' CONTRIBUTIONS}

All authors equally contributed to the writing and revision of this paper.

\section{REFERENCES}

1. Caminos JE, Nogueiras R, Gaytan F, Pineda R, Gonzalez CR, Barreiro ML, et al. Novel expression and direct effects of adiponectin in the rat testis. Endocrinology. 2008;149(7):3390-3402. doi: 10.1210/en.2007-1582 pmid: 18403483

2. Moran LJ, Pasquali R, Teede HJ, Hoeger KM, Norman RJ. Treatment of obesity in polycystic ovary syndrome: a position statement of the Androgen Excess and Polycystic Ovary Syndrome Society. Fertil Steril. 2009;92(6):19661982. doi: 10.1016/j.fertnstert.2008.09.018 pmid: 19062007

3. Thomson RL, Buckley JD, Noakes M, Clifton PM, Norman RJ, Brinkworth GD. The effect of a hypocaloric diet with and without exercise training on body composition, cardiometabolic risk profile, and reproductive function in overweight and obese women with polycystic ovary syndrome. J Clin Endocrinol Metab. 2008;93(9):3373-3380. doi: 10.1210/jc.2008-0751 pmid: 18583464

4. Ehrmann DA. Polycystic ovary syndrome. N Engl J Med. 2005;352(12):1223-1236. doi: 10.1056/NEJMra041536 pmid: 15788499

5. van Santbrink EJ, Fauser BC. Ovulation induction in normogonadotropic anovulation (PCOS). Best Pract Res Clin Endocrinol Metab. 2006;20(2):261-270. doi: 10.1016/j.beem.2006.03.002 pmid: 16772156

6. Dikmen A, Ergenoglu AM, Yeniel AO, Dilsiz OY, Ercan G, Yilmaz H. Evaluation of glycemic and oxidative/antioxidative status in the estradiol valerate-induced PCOS model of rats. Eur J Obstet Gynecol Reprod Biol. 2012;160(1):55-59. doi: 10.1016/j.ejogrb.2011.09.042 pmid: 22071112

7. Sabuncu T, Vural H, Harma M. Oxidative stress in polycystic ovary syndrome and its contribution to the risk of cardiovascular disease. Clin Chem. 2001;34(5):407-413. doi: 10.1016/S0009-9120(01)00245-4

8. Cohen G, Riahi Y, Sunda V, Deplano S, Chatgilialoglu C, Ferreri C, et al. Signaling properties of 4hydroxyalkenals formed by lipid peroxidation in diabetes. Free Radic Biol Med. 2013;65:978-987. doi: 10.1016/j.freeradbiomed.2013.08.163 pmid: 23973638

9. Setyaningsih Y, Husodo AH, Astuti I. Detection of urinary 8-hydroxydeoxyguanosine (8-OHdG) levels as a biomarker of oxidative DNA damage among home industry workers exposed to chromium. Procedia Environ Sci. 2015;23:290-296. doi: 10.1016/j.proenv.2015.01.043

10. Spritzer MD, Ibler E, Inglis W, Curtis MG. Testosterone and social isolation influence adult neurogenesis in the dentate gyrus of male rats. Neuroscience. 2011;195:180-190. doi: 10.1016/j.neuroscience.2011.08.034 pmid: 21875652

11. Rafiei S, Bazyar Y, Edalatmanesh MA. Effect of gallic acid and endurance exercise training on BDNF in a model of hippocampal degeneration. Shefaye KhatamShefaye Khatam. 2016;4(1):1-6. doi: 10.18869/acadpub.shefa.4.1.1 
12. Tahmasebi F, Movahedin M, Mazaheri Z. Poly cystic ovary model as an elevated oxidative stress factor. $J$ Mazandaran Univ Med Sci. 2015;25(127):82-91.

13. Malek-Mohammadi R, Roghani M, Salami M. The effect of aqueous extracts of Melissa officinalis on the oxidative stress indices in the midbrain tissue. Feyz. 2015;19(1):8-14.

14. Sadoughi SD. Investigation the effect of curcumin on the hormones of pituitary-ovarian axis in Alloxan-induced diabetic rats. J Ardabil Univ Med Sci. 2016;16(4):441-451.

15. Duleba AJ. Medical management of metabolic dysfunction in PCOS. Steroids. 2012;77(4):306-311. doi: 10.1016/j.steroids.2011.11.014 pmid: 22182833

16. Norouzi T, Ghatreh Samani K, Amini SA. Compare the effects of atorvastatin and omega-3 on index of lipid oxidation in patients with polycystic ovary syndrome. J Shahrekord Univ Med Sci. 2016;18(1):36-44.

17. Petrikova J, Lazurova I. Ovarian failure and polycystic ovary syndrome. Autoimmun Rev. 2012;11(6-7):A471-478. doi: 10.1016/j.autrev.2011.11.010 pmid: 22154711

18. Maharaj A. Polycystic ovary syndrome. JEMDSA. 2009;14(2):86-95. doi: 10.1080/22201009.2009.10872199

19. Lakhani K, Prelevic GM, Seifalian AM, Atiomo WU, Hardiman P. Polycystic ovary syndrome, diabetes and cardiovascular disease: risks and risk factors. J Obstet Gynaecol. 2004;24(6):613-621. doi: 10.1080/01443610400007810 pmid: 16147598

20. Azziz R, Carmina E, Dewailly D, Diamanti-Kandarakis E, Escobar-Morreale HF, Futterweit W, et al. Positions statement: criteria for defining polycystic ovary syndrome as a predominantly hyperandrogenic syndrome: an Androgen Excess Society guideline. J Clin Endocrinol Metab. 2006;91(11):4237-4245. doi: 10.1210/jc.2006-0178 pmid: 16940456

21. Moran LJ, Ranasinha S, Zoungas S, McNaughton SA, Brown WJ, Teede HJ. The contribution of diet, physical activity and sedentary behaviour to body mass index in women with and without polycystic ovary syndrome. Hum Reprod. 2013;28(8):2276-2283. doi: 10.1093/humrep/det256 pmid: 23771201

22. Murri M, Luque-Ramirez M, Insenser M, Ojeda-Ojeda M, Escobar-Morreale HF. Circulating markers of oxidative stress and polycystic ovary syndrome (PCOS): a systematic review and meta-analysis. Hum Reprod Update. 2013;19(3):268-288. doi: 10.1093/humupd/dms059 pmid: 23303572

23. Harrison CL, Lombard CB, Moran LJ, Teede HJ. Exercise therapy in polycystic ovary syndrome: a systematic review. Hum Reprod Update. 2011;17(2):171-183. doi: 10.1093/humupd/dmq045 pmid: 20833639

24. Wu C, Lin F, Qiu S, Jiang Z. The characterization of obese polycystic ovary syndrome rat model suitable for exercise intervention. PLoS One. 2014;9(6):e99155. doi: 10.1371/journal.pone.0099155 pmid: 24905232

25. Al-Nozha O, Habib F, Mojaddidi M, El-Bab MF. Body weight reduction and metformin: Roles in polycystic ovary syndrome. Pathophysiology. 2013;20(2):131-137. doi: 10.1016/j.pathophys.2013.03.002 pmid: 23608322

26. Miri M, Karimi Jashni H, Alipour F. Effect of exercise intensity on weight changes and sexual hormones (androstenedione and free testosterone) in female rats with estradiol valerate-induced PCOS. J Ovarian Res. 2014;7:37. doi: 10.1186/1757-2215-7-37 pmid: 24708600 